\title{
Hepatitis B virus infection is not associated with fatty liver disease: Evidence from a cohort study and functional analysis
}

\author{
BINGQIAN WANG, WENNA LI, HEZHI FANG and HUAIBIN ZHOU
}

\begin{abstract}
Key Laboratory of Laboratory Medicine, Ministry of Education, Zhejiang Provincial Key Laboratory of Medical Genetics, College of Laboratory Medicine and Life Sciences, Wenzhou Medical University, Wenzhou, Zhejiang 325035, P.R. China
\end{abstract}

Received February 7, 2018; Accepted October 1, 2018

DOI: $10.3892 / \mathrm{mmr} .2018 .9619$

\begin{abstract}
Chronic hepatitis B virus (HBV) infection has been reported to be associated with the prevalence of non-alcoholic fatty liver disease (NAFLD). However, the present study demonstrated that the incidence of fatty liver disease in HBV-infected subjects $(16 / 152,10.5 \%)$ was not significantly different from in non-HBV-infected subjects $(292 / 1,714,17 \%)$, following adjustment for age (odds ratio $=0.656$; $95 \%$ confidence interval=0.379-1.134; $\mathrm{P}=0.131$ ). Hepatitis $\mathrm{B}$ protein $\mathrm{X}$ (HBx) is considered a key regulator in HBV infection and several studies have confirmed that HBx serves a pivotal role in the process of fatty liver disease. In the present study, it was demonstrated that HBx-expressing cells exhibited increased mitochondrial membrane potential, ATP generation, and endogenous mitochondrial respiration. In addition, higher levels of mitochondrial reactive oxygen species (ROS) were detected in HBx-expressing cells compared with in control cells. Increased ROS production may contribute to increased lipid droplet formation in HBx-expressing cells, whereas the removal of ROS with $\mathrm{N}$-acetylcysteine may decrease the accumulation of lipid droplets in a time-dependent manner. In conclusion, the present findings indicated that HBV, and perhaps more specifically $\mathrm{HBx}$, was not a protective factor against NAFLD. HBx may function as a risk factor for fatty liver disease, based on the findings of the present functional study; however, further studies are required to clarify the effects of HBx on hepatic steatosis.
\end{abstract}

Correspondence to: Dr Huaibin Zhou, Key Laboratory of Laboratory Medicine, Ministry of Education, Zhejiang Provincial Key Laboratory of Medical Genetics, College of Laboratory Medicine and Life Sciences, Wenzhou Medical University, 1 Central Northern Road, University Town, Wenzhou, Zhejiang 325035, P.R. China

E-mail: zhouhb519@163.com

Key words: hepatitis $\mathrm{B}$ virus/hepatitis $\mathrm{B}$ protein $\mathrm{X}$, fatty liver disease, non-alcoholic fatty liver disease, reactive oxygen species, lipid droplet

\section{Introduction}

Fatty liver disease, particularly non-alcoholic fatty liver disease (NAFLD), is a major subtype of liver disease (1). Fatty liver disease is characterized by lipid accumulation in the liver and is closely associated with mitochondrial abnormality (2). Generally, abnormal mitochondrial function and increased mitochondrial reactive oxygen species (ROS) affect fatty liver disease via impaired insulin sensitivity (3). Elevated mitochondrial ROS production can also cause steatohepatitis, due to fatty acid oxidation disorder, which is an increased inflammatory response in the liver (4). Therefore, mitochondrial-targeted antioxidants are considered potential, effective therapeutic compounds for the treatment of fatty liver disease (5).

Millions of people worldwide suffer from chronic hepatitis B virus (HBV) infection (6). Chronic HBV infection affects numerous organ systems and is associated with several human diseases (7). The pathogenesis of liver disease is the most studied aspect of HBV infection, since the liver is the most affected organ in HBV carriers (8); however, the underlying molecular mechanisms by which HBV affects the liver remain poorly understood. Recently, a case-control study involving different populations indicated that patients with HBV infection suffer from fewer fatty liver diseases, including NAFLD (9); however, some studies hold the opposite view, that HBV infection increases the prevalence of NAFLD $(10,11)$.

The HBV genome is a partially double-stranded, circular DNA molecule that encodes four main HBV proteins: Proteins C, X, P and S. Of these, hepatitis B protein X (HBx) is a multifunctional regulator involved in cellular signal transduction pathways and gene transcription (12). HBV infection and HBx expression in cells affect cellular processes, including cell growth, cell cycle progression and apoptosis, thus indicating a pivotal role for HBx in HBV infection (13). Therefore, it is not surprising that HBV infection is associated with NAFLD (14). HBx was originally detected in the cytoplasm and nucleus (12), and due to its transcriptional function, $\mathrm{HBx}$ can alter the gene expression of the host cell (15). In mitochondria, HBx has been reported serve as an enhancer of mitochondrial function by direct interaction with the mitochondrial respiratory chain complex subunit (15). However, HBx may also contribute to mitochondrial function via increased fission and mitophagy (16). These conflicting 
findings increase the difficulty of analyzing the mitochondrial role in $\mathrm{HBV} / \mathrm{HBx}$-associated fatty liver disease, specifically NAFLD. While there is no consensus in the literature regarding the association between HBV and protection against NAFLD, to understand the effects of HBV infection on NAFLD, the present study conducted a large-scale case-control study on Han Chinese individuals. In addition, a functional study using $\mathrm{HBx}$-expressing hepatocytes was performed to validate the effect of HBx-mediated HBV infection on NAFLD.

\section{Patients and methods}

Subjects. A total of 1,882 female subjects that underwent comprehensive health examination were recruited at the First Affiliated Hospital of Wenzhou Medical University (Wenzhou, China) between October 2011 and March 2014 (mean age \pm standard deviation, $46.42 \pm 10.291$ years; median age, 45 years; age range 19-83 years). Since subjects with hepatitis $\mathrm{C}$ virus (HCV) have alterations in lipid metabolism (17), HCV infection was tested by Roche Cobas7 Amplicor HCV Monitor Test, version 2.0, (Roche Molecular Diagnostics, Pleasanton, CA, USA). Subsequently, 16 subjects with chronic HCV infection were excluded from the present study; the response rate for the enrolled subjects was thus $99.1 \%$ (1,866/1,882; Fig. 1). By ultrasonography, using the Hitachi VISION Preirus (Hitachi, Ltd., Tokyo, Japan), according to four ultrasound criteria (liver brightness, deep attenuation, hepatorenal echo contrast and vascular blurring), 308 and 1,558 subjects were diagnosed with fatty liver and non-fatty liver disease, respectively. The presence of hepatitis B virus antigen (HBsAg) was determined in all enrolled subjects using the Roche Cobas 6800/8800 Systems (Roche Diagnostics). As demonstrated in Fig. 1, 16 subjects from the fatty liver group and 136 subjects from the non-fatty liver group were considered to have HBV, due to the presence of HBsAg (9). Informed consent was obtained from all subjects and this study was approved by the Ethical Committee of Wenzhou Medical University.

Cells and culture conditions. The hepatocyte cell lines Huh7 and MIHA were purchased from the Cell Resource Center, Chinese Academy of Medical Sciences (Beijing, China); the two cell types are widely used for the study of HBV infection $(18,19)$. All cells were cultured in high-glucose Dulbecco's modified Eagle's medium (Gibco; Thermo Fisher Scientific, Inc., Waltham, MA, USA) containing $10 \%$ fetal bovine serum (Sigma-Aldrich; Merck KGaA, Darmstadt, Germany) and were maintained at $37^{\circ} \mathrm{C}$ in a humidified atmosphere containing $5 \% \mathrm{CO}_{2}$.

Plasmid infection. The coding sequence of HBx with a c-Myc tag at the $\mathrm{C}$ terminus (Gene ID: 944566) was synthesized and cloned into a pCDH plasmid (donated by Dr. Zhenfen Chi, Beijing Institute of Genomics). 293 T cells were from Cell Resource Center, Chinese Academy of Medical Sciences (Shanghai, China). Briefly, 293T cells $\left(\sim 1 \times 10^{6}\right)$ were co-transfected with $1 \mu \mathrm{g}$ pCDH with or without $\mathrm{HBx}, 1 \mu \mathrm{g}$ psPAX2, and $0.5 \mu \mathrm{g}$ PMD2G (donated by Dr. Zhenfen Chi; 2:2:1) for $72 \mathrm{~h}$. Culture medium with packaged lentivirus was used to infect Huh7 and MIHA cells for $24 \mathrm{~h}$. Cells expressing HBx were selected using $4 \mu \mathrm{g} / \mathrm{ml}$ puromycin (Sigma-Aldrich; Merck KGaA). An empty plasmid was used as a control.

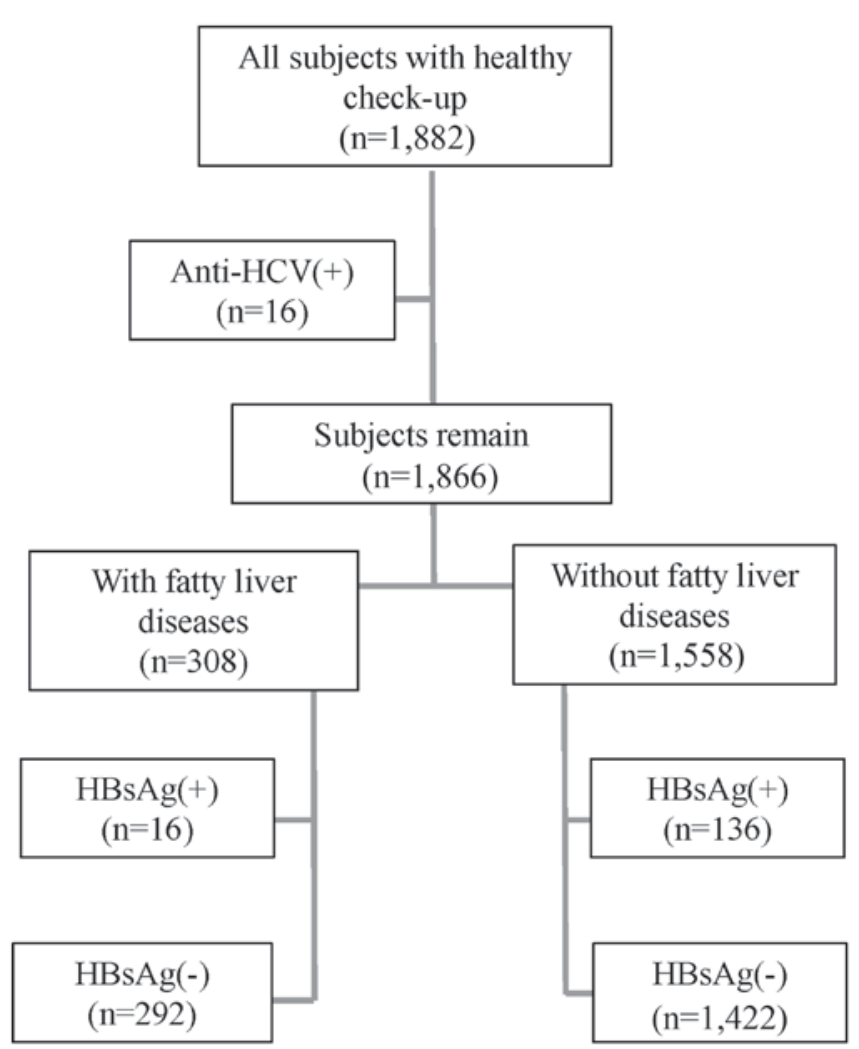

Figure 1. Cohort study flow chart. HBV, hepatitis B virus; HBsAg, hepatitis B antigen; $\mathrm{HCV}$, hepatitis $\mathrm{C}$ virus.

Antibodies and immunoblotting. Protein was extracted from whole cell lysates using radioimmunoprecipitation assay lysis buffer (Cell Signaling Technology, Inc., Danvers, MA, USA). Protein concentration was quantified using Pierce BCA Protein Assay kit (Thermo Fisher Scientific, Inc.). Samples containing $30 \mu \mathrm{g}$ proteins were electrophoresed through $12 \%$ Sodium dodecyl sulfate polyacrylamide gel electrophoresis (SDS-PAGE), and transferred onto PVDF membranes with $0.2 \mu \mathrm{m}$ pore size (Thermo Fisher Scientific, Inc.), the membrane was blocked with tris buffered saline with 1\% Tween 20 (TBST) buffer containing 10\% non-fat milk at room temperature for $1 \mathrm{~h}$. Proteins were probed with anti-HBx antibody (ab2741; 1:1,000; Abcam, Cambridge, UK) or anti- $\beta$-actin at $4^{\circ} \mathrm{C}$ for $16 \mathrm{~h}(3700 ; 1: 1,000$; Cell Signaling Technology, Inc., Danvers, MA, USA). Horseradish peroxidase conjugated anti mouse IgG (4410; 1:2,000; Cell Signaling Technology, Inc.) was used as secondary antibodies and incubated for $1 \mathrm{~h}$ at room temperature. Signals were detected with Super Signal West Pico chemiluminescent substrate (Thermo Fisher Scientific, Inc.). Integrated optical density was semi-quantified using a Gel-Pro Analyzer 4.0 (Media Cybernetics, Inc., Rockville, MD, USA).

Immunofluorescence staining. Cells $\left(3 \times 10^{5}\right)$ were treated with 100 nM MitoTracker Red (Invitrogen; Thermo Fisher Scientific, Inc.) for $10 \mathrm{~min}$ at $37^{\circ} \mathrm{C}$ and were fixed with $4 \%$ paraformaldehyde for $30 \mathrm{~min}$ in room temperature. After treatment with $0.2 \%$ Triton $\mathrm{X}-100$ for $3 \mathrm{~min}$, cells were incubated overnight with anti-c-Myc antibody (2276s; 1:1,000; Cell Signaling Technology, Inc.). Subsequently, cells were washed with PBS and incubated with an Alexa Fluor ${ }^{\circledR}$ 488-labeled 
Table I. Factors associated with HBV and non-HBV subjects.

\begin{tabular}{lccc}
\hline Characteristic & HBsAg $^{+}$ & HBsAg $^{-}$ & P-value $^{\mathrm{a}}$ \\
\hline Number & 152 & 1,714 & - \\
Age (years) & $45.1 \pm 8.7$ & $46.5 \pm 10.4$ & 0.052 \\
BMI (kg/m $\left.{ }^{2}\right)$ & $23.94 \pm 3.16$ & $22.85 \pm 3.07$ & $<0.001$ \\
SBP $(\mathrm{mmHg})$ & $118.6 \pm 14.9$ & $115.9 \pm 14.9$ & 0.010 \\
DBP (mmHg) & $78.7 \pm 9.7$ & $75.3 \pm 9.3$ & $<0.001$ \\
ALT (U/I) & $37.1 \pm 41.0$ & $19.8 \pm 17.6$ & $<0.001$ \\
AST (U/I) & $30.9 \pm 29.8$ & $20.5 \pm 10.2$ & $<0.001$ \\
GGT (U/I) & $36.2 \pm 64.2$ & $21.2 \pm 19.6$ & $<0.001$ \\
Glucose (mg/dl) & $5.6 \pm 1.1$ & $5.5 \pm 1.0$ & 0.363 \\
Total cholesterol (mg/dl) & $4.7 \pm 0.8$ & $4.9 \pm 0.9$ & 0.261 \\
LDL-C (mg/dl) & $2.8 \pm 0.7$ & $2.7 \pm 0.8$ & 0.094 \\
HDL-C (mg/dl) & $1.4 \pm 0.4$ & $1.5 \pm 0.3$ & $\mathrm{P}<0.001$ \\
Triglycerides (mg/dl) & $1.4 \pm 0.8$ & $1.2 \pm 1.0$ & 0.061
\end{tabular}

${ }^{a}$ Adjusted for age. ALT, alanine aminotransferase; AST, aspartate aminotransferase; BMI, body mass index; DBP, diastolic blood pressure; GGT, $\gamma$-glutamyl transferase; HDL-C, high-density lipoprotein cholesterol; LDL-C, low-density lipoprotein cholesterol; SBP systolic blood pressure.

immunoglobulin secondary antibody (9854; 1:250; Cell Signaling Technology, Inc.) for $45 \mathrm{~min}$ at room temperature. A final incubation was performed to stain the cells with DAPI (Beyotime Institute of Biotechnology, Haimen, China) for $5 \mathrm{~min}$ at room temperature. Images were captured using a confocal laser microscope at a magnification x400 (Nikon Corporation, Tokyo, Japan).

Measurement of endogenous oxygen consumption. The endogenous oxygen consumption of intact cells was determined using a Clark-type oxygen electrode (Oroboros Instruments Corp., Innsbruck, Austria), as described previously (20). After recording basal respiration, oligomycin (an ATP synthase inhibitor; $0.2 \mu \mathrm{g} / \mathrm{ml}$; Sigma-Aldrich; Merck KGaA) was added to measure uncoupling respiration, and carbonyl cyanide m-chlorophenyl hydrazone (CCCP; a mitochondrial oxidative phosphorylation uncoupling agent; $0.1 \mu \mathrm{M}$; Sigma-Aldrich; Merck $\mathrm{KGaA}$ ) was added to measure maximum respiration.

ATP, mitochondrial membrane potential (MMP) and ROS measurements. ATP was measured using an ATP determination kit (A22066; Molecular Probes; Thermo Fisher Scientific, Inc.), according to the manufacturer's protocol. Briefly, cells were grown in 6 -well plates to $\sim 80 \%$ confluence. Cells $\left(\sim 1 \times 10^{6}\right)$ were then washed with cold PBS and boiled in $100 \mu \mathrm{l}$ boiling buffer (100 mM Tris, 4 mM EDTA, adjusted to $\mathrm{pH} 7.75$ with acetic acid) for $90 \mathrm{sec}$. Supernatants were retrieved by centrifugation at $10,000 \mathrm{x}$ g for $1 \mathrm{~min}$. ATP content was determined by measuring the luminescence of supernatants mixed with luciferase assay buffer and luminescence measured at $\sim 560 \mathrm{~nm}$ using a Varioskan ${ }^{\mathrm{TM}}$ Flash Multimode Reader (both Thermo Fisher Scientific, Inc.).

Mitochondrial ROS levels were measured as described previously (21). Briefly, $1 \times 10^{6}$ cells were washed in Hank's buffered salt solution (HBSS; Sigma-Aldrich; Merck KGaA) resuspended in HBSS containing $5 \mu \mathrm{M}$ Mito SOX (Molecular Probes; Thermo Fisher Scientific, Inc.), and incubated at $37^{\circ} \mathrm{C}$ for $15 \mathrm{~min}$. Cells were then washed twice with HBSS, and fluorescence with excitation/emission at 510/580 nm was recorded using a Varioskan ${ }^{\mathrm{TM}}$ Flash Multimode Reader. To measure the ROS level in cells with NAC, Huh7 and MIHA cells were cultured with $10 \mathrm{mM} \mathrm{NAC}$ for $48 \mathrm{~h}$ at $37^{\circ} \mathrm{C}$ in a cell culture incubator (Sigma-Aldrich; Merck KGaA) for 48 h. MMP was determined using the cationic fluorescent redistribution dye TMRM (Thermo Fisher Scientific, Inc.) as described previously (22). ATP, MMP and ROS signals were normalized by the protein concentration measured with the Pierce BCA Protein Assay kit.

Oil Red $O(O R O)$ staining. Cells $\left(5 \times 10^{5}\right)$ were fixed with $4 \%$ formaldehyde at room temperature for $25 \mathrm{~min}$, and were stained with freshly diluted ORO reagent (Sangon Biotech Co., Ltd., Shanghai, China; diluted distilled water:Oil red O 4:6) for $20 \mathrm{~min}$ at room temperature. Subsequently, cells were stained with $0.5 \%$ hematoxylin (Sangon Biotech Co., Ltd.) for 5 min at room temperature and washed with PBS twice. Lipid accumulation in the cells was observed using an inverted phase contrast optical microscope at magnification, x200 (ECLIPSE Ti-S; Nikon Corporation). To measure lipid droplet accumulation in cell with NAC treatment, Huh7 and MIHA cells were cultured with $10 \mathrm{~nm} \mathrm{NAC}$ for $48 \mathrm{~h}$ at $37^{\circ} \mathrm{C}$ in a cell culture incubator before ORO staining.

Statistical analysis. Clinical data are presented as means \pm standard deviation. Categorical variables were compared using the $\chi^{2}$ test. The association between fatty liver and HBV infection was adjusted for age using the logistic regression. Data from cell models are presented as the means \pm standard error of the mean. To evaluate cell model data, an independent Student's t-test was used. $\mathrm{P}<0.05$ was considered to indicate a statistically significant difference. All statistical analyses were performed using SPSS 21.0 (IBM Corp., Armonk, NY, USA).

\section{Results}

$H B V$ infection is not associated with fatty liver disease occurrence. Only $30 \%$ of women in China consume alcohol, and few of them have at least one drink per day (23). Correspondingly, in women with fatty liver disease, few are diagnosed with alcoholic fatty liver disease (24). Therefore, to assess the risk of HBV infection in NAFLD, women undergoing health check-ups were recruited to the present study. The clinical characteristics in subjects with or without HBV infection were initially analyzed. As demonstrated in Table I, HBV-infected subjects had significantly higher levels of alanine aminotransferase, aspartate aminotransferase and $\gamma$-glutamyl transferase than HBV-free subjects, which indicated that hepatocyte injury was present in HBV carriers. Additionally, although the levels of high-density lipoprotein, a protective factor against fatty liver disease, were significantly lower in HBV-infected patients compared with in HBV-free subjects, other fatty liver disease-associated factors, such as total cholesterol, low-density lipoprotein and triglycerides, were not significantly affected by HBV (Table I). Furthermore, the incidence of fatty liver in 
Table II. Unadjusted prevalence and age-adjusted odds ratios for fatty liver by HBV status.

\begin{tabular}{lccccr}
\hline & & \multicolumn{3}{c}{ Fatty liver } \\
\cline { 3 - 5 } Group & Total number & Total cases (prevalence \%) & OR & $95 \%$ CI & P-value $^{\text {a }}$ \\
\hline $\mathrm{HBsAg}^{-}$ & 1,714 & $292(17.0)$ & 1 & \\
$\mathrm{HBsAg}^{+}$ & 152 & $16(10.5)$ & 0.656 & $0.379-1.134$ & 0.131 \\
\hline
\end{tabular}

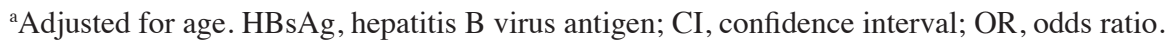

A

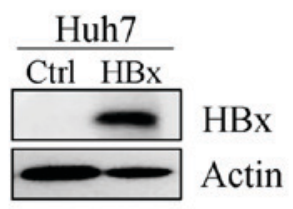

C

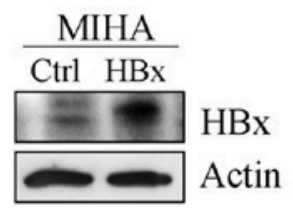

$\mathrm{B}$

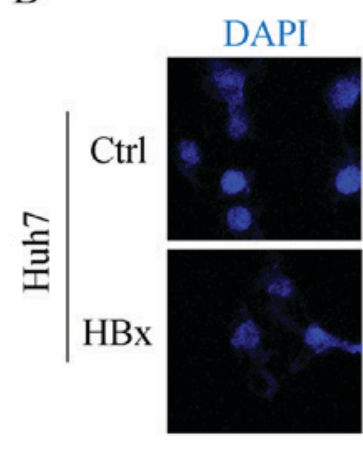

$\mathrm{D}$

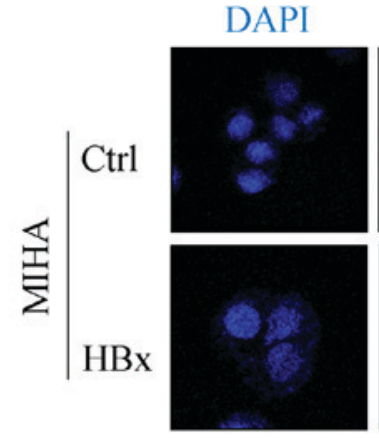

$\mathrm{HBx}$

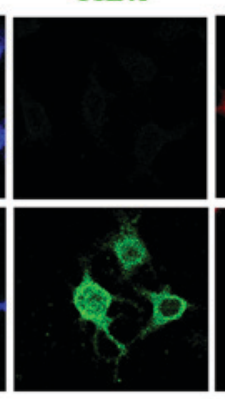

$\mathrm{HBx}$
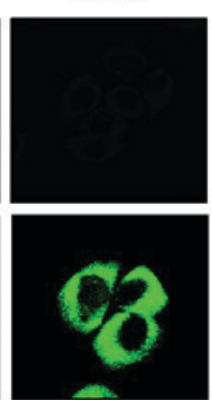

Mito

Tracker

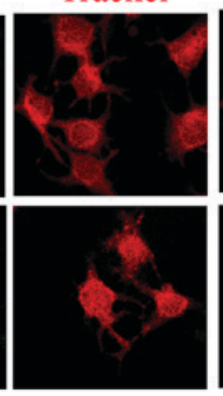

Mito
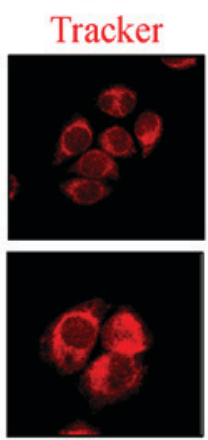

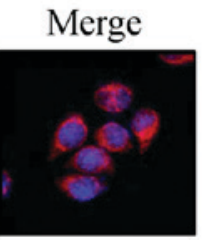

Merge
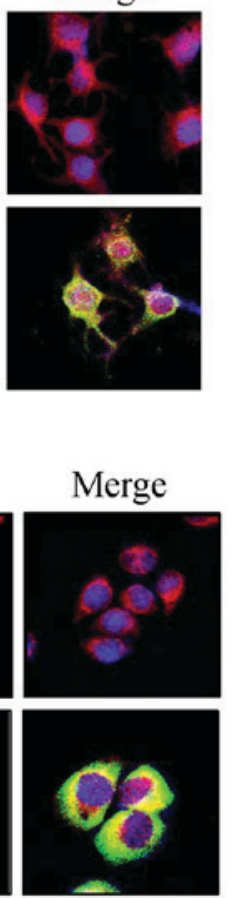

Figure 2. HBx localizes to the mitochondria in Huh7 and MIHA cells expressing HBx. Representative (A) immunoblotting and (B) immunofluorescence images of HBx protein in Huh7 cells, and (C) immunoblotting and (D) immunofluorescence images of HBx protein in MIHA cells. Cells were probed with anti-c-Myc (green) and a mitochondrial marker (MitoTracker Red). Magnification, x400. HBx, hepatitis B protein X.

HBV-infected subjects $(16 / 152,10.5 \%)$ was not significantly different from that in HBV-free subjects $(292 / 1,714,17 \%)$ when adjusted for age (Table II). These findings indicated that HBV was not associated with a decreased incidence of NAFLD.

Expression of HBx protein in Huh7 and MIHA cells increases mitochondrial function. $\mathrm{HBx}$ is the major HBV component that contributes to steatohepatitis (25). To examine if and how HBV affects the occurrence of NAFLD, the HBx protein was expressed in Huh7 and MIHA cell lines. Mitochondrial localization of HBx was observed in Huh7 (Fig. 2A and B) and MIHA cells (Fig. 2C and D), which confirmed that HBx targeted the mitochondria in the cytoplasm. Subsequently, whether $\mathrm{HBx}$ affects mitochondrial function was investigated by measuring mitochondrial respiration, MMP and ATP content. As shown in Fig. 3A and B, endogenous basal mitochondrial respiration in cells expressing HBx was significantly increased compared with in the control cells. In addition, lowest mitochondrial respiration (uncoupled respiration) in the presence of oligomycin $(0.2 \mu \mathrm{g} / \mathrm{ml})$ and maximal mitochondrial respiration in the presence of CCCP $(0.1 \mu \mathrm{M})$ were significantly increased in the two HBx-expressing cells compared with the control cells (Fig. 3A and B). To further clarify the alterations of mitochondrial function in cells expressing $\mathrm{HBx}$, the MMP and ATP content were investigated. As shown in Fig. 3C and D, MMP and ATP content were significantly increased in HBx-expressing Huh7 and MIHA cells compared with in control cells. Although the underlying mechanism is not currently known, the present results indicated that HBx may be associated with increased mitochondrial function.

HBx mediates lipid droplet accumulation in Huh7 and MIHA cells by altering mitochondrial ROS levels. Excessive mitochondrial ROS production serves an important role in the development of NAFLD (26). Therefore, mitochondrial ROS levels in Huh7 and MIHA cells with or without HBx were investigated. As shown in Fig. 4A and B, mitochondrial ROS levels were significantly increased in Huh7 and MIHA cells 
A
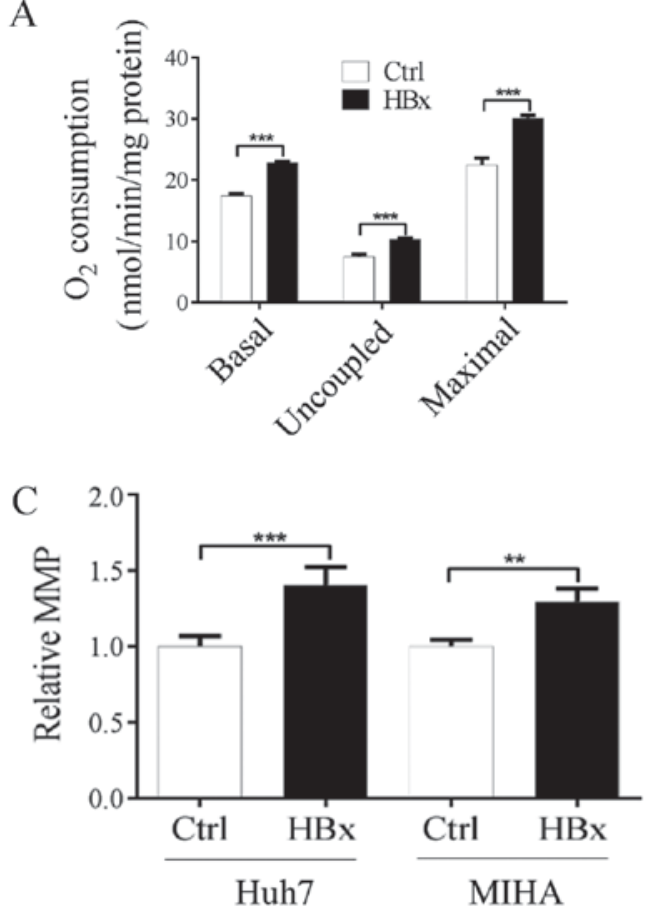

B

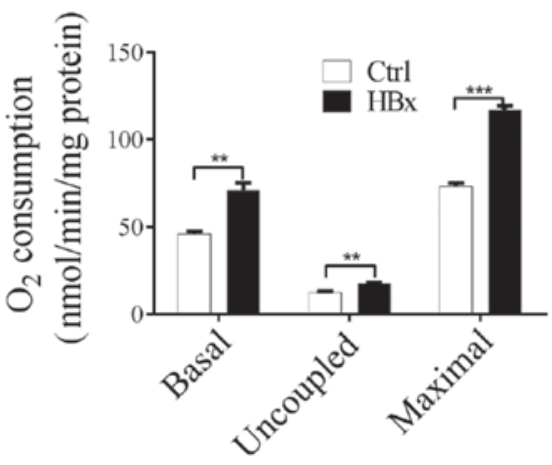

$\mathrm{D}$

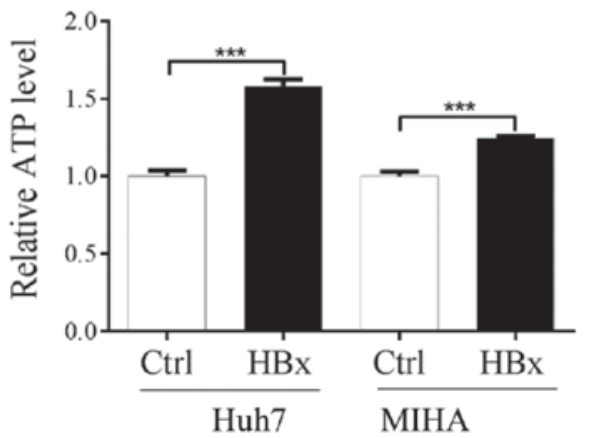

Figure 3. HBx protein increases mitochondrial function in Huh7 and MIHA cells expressing HBx. Analysis of the oxygen consumption rate in (A) Huh7 and (B) MIHA cells, with or without HBx expression, using a Clark-type oxygen electrode. Basal, basal mitochondrial respiration; uncoupled, respiration in the presence of oligomycin $(0.2 \mu \mathrm{g} / \mathrm{ml})$; maximal, respiration in the presence of carbonyl cyanide m-chlorophenyl hydrazone (0.1 $\mu \mathrm{M})$. (C) Relative MMP levels and (D) total ATP generation was determined in Huh7 (Ctrl and Huh7-HBx) and MIHA (Ctrl and MIHA-HBx) cells. Data are presented as the means \pm standard deviation $(\mathrm{n} \geq 3) .{ }^{* *} \mathrm{P}<0.01,{ }^{* * *} \mathrm{P}<0.001$. ATP, adenosine triphosphate; HBx, hepatitis B protein X; MMP, matrix metalloproteinase.

expressing HBx compared with in control cells without HBx, and this effect was reversed by treating cells with $\mathrm{N}$-acetylcysteine for up to $48 \mathrm{~h}$. Furthermore, it was demonstrated that Huh7 and MIHA cells expressing HBx exhibit increased fatty droplet formation compared with in the control cells (Fig. 4C and D), which further confirmed that HBV infection may increase the pathogenesis of fatty liver disease. Alternatively, increased ROS production in hepatocytes expressing HBx may promote lipid droplet accumulation, and this effect was reversed by treating the cells with a ROS scavenger in a time-dependent manner (Fig. 4C and D). Therefore, the present findings indicated that, $\mathrm{HBV}$, or at least the HBx protein, may not be a protective factor against NAFLD; however, this requires further validation.

\section{Discussion}

Viral infections, such as chronic HBV or HCV, can cause a multitude of liver diseases, including hepatic fibrosis, cirrhosis and liver cancer $(27,28)$. Unlike HCV, conflicting conclusions regarding the association between HBV infection and fatty liver disease have been reported in different populations (29-31). The prevalence of NAFLD is $28.3 \%$ in Hong Kong (age range, 18-70) (31) and $43.9 \%$ in Taiwan (mean age, 51.9 years), whereas in patients with $\mathrm{HBV}$, the prevalence of NAFLD decreases to 13.5 and $38.9 \%$ in Hong Kong and Taiwan, respectively $(31,32)$. However, in Hong Kong, a significant difference in the prevalence of NAFLD between HBV-infected individuals and controls was detected only in the population aged between 40 and 59 years. Conversely, whereas HBV infection has been reported to be negatively associated with NAFLD in patients $>40$ years, the same association is not true in younger patients (32). Despite this contradiction, a recent large cohort study in Korea supported the hypothesis that HBV is a protective factor against NAFLD (9). In the present study, female patients with fatty liver disease were recruited, in order to study the association between HBV infection and fatty liver disease. The majority of women in China do not consume alcohol, and few have a history of daily consumption, due to traditional Chinese culture; therefore, few female patients with fatty liver disease are diagnosed with alcoholic fatty liver disease. The prevalence of female fatty liver disease in the present study (24.8\% in 50-59 year old age group) is similar to that reported for Chinese female patients with NAFLD in the same age range (22.8\%) (33). Studying the association between female patients with fatty liver disease and HBV infection may provide important information regarding the effects of HBV infection on NAFLD. Since age has been demonstrated to be a major contributor to HBV-associated NAFLD $(31,32)$, logistic regression analysis was applied to adjust for the risks associated with age and HBV infection in fatty liver disease. Although the prevalence of fatty liver disease was lower in patients with HBV (10.5\%) compared with in HBV-free patients (17\%), this finding was not significant. These findings suggested that HBV may not be a protective factor against fatty liver disease or NAFLD in mainland China.

The present epidemiological data is preliminary and limitations exist. Factors such as anti-viral usage, the stage of $\mathrm{HBV}$ infection and infection history in HBV-infected patients were not considered. Furthermore, information regarding alcohol consumption and drug administration in the enrolled subjects were not included. However, Joo et al (9) reported similar limitations, but the large sample size used in their study 
A

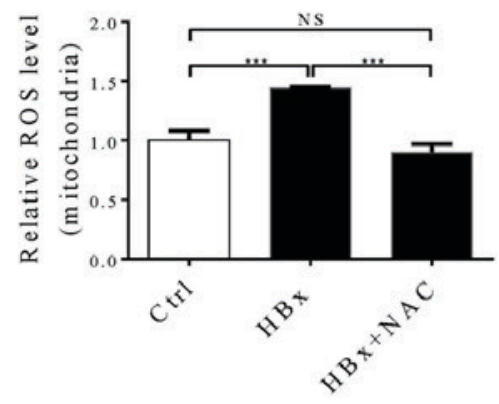

C

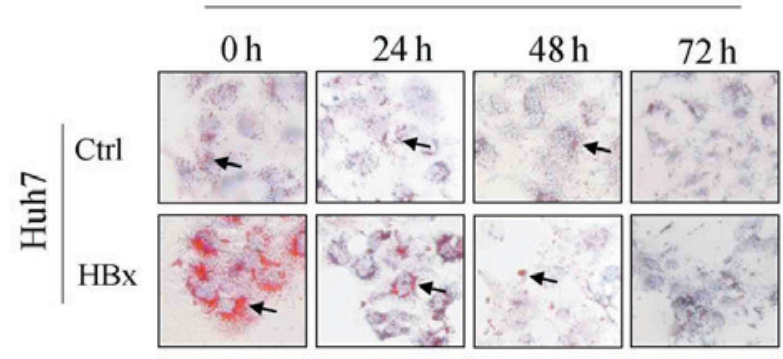

B

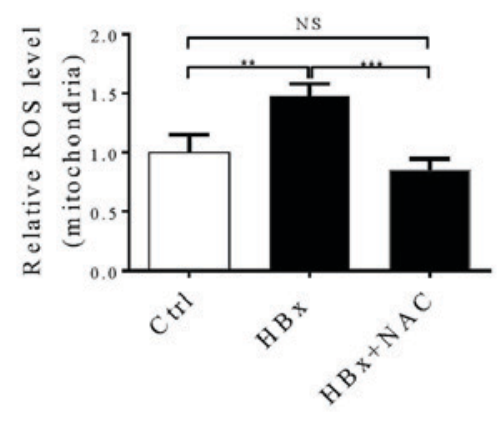

D

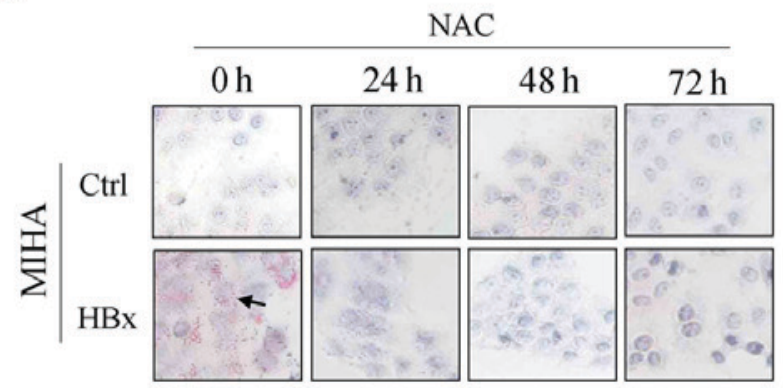

Figure 4. ROS mediate lipid droplet accumulation in Huh7 and MIHA cells expressing HBx. Relative mitochondrial ROS levels in (A) Huh7 (Ctrl and Huh7-HBx) and (B) MIHA (Ctrl and MIHA-HBx) cells. (C) Huh7 (Ctrl and Huh7-HBx) and (D) MIHA (Ctrl and MIHA-HBx) cells were cultured with or without 2 mM NAC for 24, 48, and 72 h. Lipid droplets (shown with black arrows) in liver cells were examined by Oil Red O staining and were observed under an inverted phase contrast microscope. Magnification, $\mathrm{x} 200$. Data are presented as the means \pm standard deviation ( $\mathrm{n} \geq 3$ ). ${ }^{* *} \mathrm{P}<0.01,{ }^{* * *} \mathrm{P}<0.001, \mathrm{NS}, \mathrm{not}$ significant; HBx, hepatitis B protein X; NAC, N-acetylcysteine; ROS, reactive oxygen species.

helped to overcome them. In the present study, the effects of $\mathrm{HBx}$ on lipid levels in the two cell lines were investigated to further evaluate the effects of $\mathrm{HBV}$, specifically $\mathrm{HBx}$, on fatty liver disease. The present results demonstrated that HBx increased the formation of lipid droplets in vitro, a key indicator of NAFLD, suggesting that HBx may serve as an inducer of NAFLD. HBx is believed to act as a promoter in hepatic lipid accumulation through $\mathrm{HBx} /$ fatty acid-binding protein $1 /$ hepatocyte nuclear factor 3- $\beta$ (HNF3 $\beta$ ), CCAAT-enhancer-binding protein $\alpha$ and peroxisome proliferator-activated receptor $\alpha$ axis in HBV-induced NAFLD (34). In addition, HBx can directly interact with liver $\mathrm{X}$ receptor (LXR) $\alpha$ to upregulate the levels of sterol regulatory element-binding protein 1 (SREBP1c) to support lipogenesis, thereby causing fatty liver disease (35). In the present study, it was demonstrated that HBx may promote lipid droplet formation via increased mitochondrial ROS levels, whereas the addition of a ROS scavenger inhibited lipid droplet formation. The underlying mechanism of ROS in lipid droplet formation and NAFLD has been widely studied (36). In addition to the $\mathrm{HBx} / \mathrm{LXR} \alpha / \mathrm{SREBP} 1$ axis, an $\mathrm{HBx} /$ mitochondrial ROS/c-Jun N-terminal kinase axis can also promote SREBP1c to support the formation of lipid droplet formation (37). Although increased generation of ROS production has been implicated in damaged mitochondria, increased mitochondrial ROS are also detected in cells with elevated aerobic metabolism (38). Consistently, our finding of increased mitochondrial function and ROS production in HBx transfected cells imply a possibility of increased electron leakage from over-activated electron transfer.

In conclusion, unlike previous studies, the present study demonstrated that HBV infection may not act as a protective factor against NAFLD in the Chinese population. Conversely, it was revealed that HBV may serve a role in the development of NAFLD in vitro via increased production of mitochondrial ROS; however, this requires further validation in future studies.

\section{Acknowledgements}

The authors thank Nianqi Hu (Wenzhou Medical University) for her assistance on data analysis of this manuscript.

\section{Funding}

The present study was supported by grants from the Chinese National Science Foundation (grant no. 31370795).

\section{Availability of data and materials}

The analyzed data sets generated during the study are available from the corresponding author on reasonable request.

\section{Authors' contributions}

HZ conceived the study, performed research and wrote the manuscript; BW and WL performed research, analyzed data and wrote the manuscript; BW conducted the statistical analysis; BW, HZ and HF analyzed data and participated in the critical discussion.

\section{Ethics approval and consent to participate}

Informed consent was obtained from all subjects, and this study was approved by the Ethical Committee of Wenzhou Medical University. 


\section{Patient consent for publication}

Not applicable.

\section{Competing interests}

The authors declare that they have no competing interests.

\section{References}

1. Benedict M and Zhang X: Non-alcoholic fatty liver disease: An expanded review. World J Hepatol 9: 715-732, 2017.

2. Gusdon AM, Song KX and Qu S: Nonalcoholic Fatty liver disease: Pathogenesis and therapeutics from a mitochondria-centric perspective. Oxid Med Cell Longev 2014: 637027, 2014.

3. Besse-Patin A and Estall JL: An intimate relationship between ROS and insulin signalling: Implications for antioxidant treatment of fatty liver disease. Int J Cell Biol 2014: 519153, 2014

4. Mello T, Materozzi M and Galli A: PPARs and mitochondrial metabolism: From NAFLD to HCC. PPAR Res 2016: 7403230, 2016.

5. Bravo E, Palleschi S, Rossi B, Napolitano M, Tiano L, D'Amore E and Botham KM: Coenzyme Q metabolism is disturbed in high fat diet-induced non-alcoholic fatty liver disease in rats. Int J Mol Sci 13: 1644-1657, 2012.

6. Schweitzer A, Horn J, Mikolajczyk RT, Krause G and Ott JJ: Estimations of worldwide prevalence of chronic hepatitis B virus infection: A systematic review of data published between 1965 and 2013. Lancet 386: 1546-1555, 2015.

7. Hsu A, Lai CL and Yuen MF: Update on the risk of hepatocellular carcinoma in chronic hepatitis B virus infection. Curr Hepat Rep 10: 106-111, 2011

8. Tu T, Budzinska MA, Shackel NA and Urban S: HBV DNA integration: Molecular Mechanisms and Clinical Implications. Viruses 9: pii: E75, 2017.

9. Joo EJ, Chang Y, Yeom JS and Ryu S: Hepatitis B virus infection and decreased risk of nonalcoholic fatty liver disease: A cohor study. Hepatology 65: 828-835, 2017.

10. He J, Zeng YL, Li W, Guo EE, Li JL, Kang Y and Shang J: Clinical study of non-alcoholic fatty liver disease and its combined the chronic HBV infection. Zhonghua Gan Zang Bing Za Zhi 25: 618-622, 2017 (In Chinese).

11. Lin HJ, Ku KL, Lin IH and Yeh CC: Naringenin attenuates hepatitis $\mathrm{B}$ virus $\mathrm{X}$ protein-induced hepatic steatosis. BMC Complement Altern Med 17: 505, 2017.

12. Bouchard MJ and Schneider RJ: The enigmatic X gene of hepatitis B virus. J Virol 78: 12725-12734, 2004.

13. Chen HY, Tang NH, Lin N, Chen ZX and Wang XZ: Hepatitis $\mathrm{B}$ virus $\mathrm{X}$ protein induces apoptosis and cell cycle deregulation through interfering with DNA repair and checkpoint responses. Hepatol Res 38: 174-182, 2008.

14. Na TY, Shin YK, Roh KJ, Kang SA, Hong I, Oh SJ, Seong JK, Park CK, Choi YL and Lee MO: Liver X receptor mediates hepatitis B virus $\mathrm{X}$ protein-induced lipogenesis in hepatitis B virus-associated hepatocellular carcinoma. Hepatology 49 : 1122-1131, 2009

15. Kim KH, Shin HJ, Kim K, Choi HM, Rhee SH, Moon HB, Kim HH, Yang US, Yu DY and Cheong J: Hepatitis B virus X protein induces hepatic steatosis via transcriptional activation of SREBP1 and PPARgamma. Gastroenterology 132: 1955-1967, 2007.

16. Kim SJ, Khan M, Quan J, Till A, Subramani S and Siddiqui A: Hepatitis B virus disrupts mitochondrial dynamics: Induces fission and mitophagy to attenuate apoptosis. PLoS Pathog 9: e1003722, 2013.

17. Valkov I, Ivanova R, Alexiev A, Antonov K and Mateva L: Association of serum lipids with hepatic steatosis, stage of liver fibrosis and viral load in chronic Hepatitis C. J Clin Diagn Res 11: OC15-OC20, 2017.

18. Chen J, Zhang W, Lin J, Wang F, Wu M, Chen C, Zheng Y, Peng X, Li J and Yuan Z: An efficient antiviral strategy for targeting hepatitis B virus genome using transcription activator-like effector nucleases. Mol Ther 22: 303-311, 2014

19. Li CH, Xu F, Chow S, Feng L, Yin D, Ng TB and Chen Y: Hepatitis B virus X protein promotes hepatocellular carcinoma transformation through interleukin- 6 activation of microRNA-21 expression. Eur J Cancer 50: 2560-2569, 2014.
20. Gong S, Peng Y, Jiang P, Wang M, Fan M, Wang X, Zhou H, Li H, Yan Q, Huang T and Guan MX: A deafness-associated tRNAHis mutation alters the mitochondrial function, ROS production and membrane potential. Nucleic Acids Res 42: 8039-8048, 2014.

21. Park JS, Sharma LK, Li H, Xiang R, Holstein D, Wu J, Lechleiter J, Naylor SL, Deng JJ, Lu J and Bai Y: A heteroplasmic, not homoplasmic, mitochondrial DNA mutation promotes tumorigenesis via alteration in reactive oxygen species generation and apoptosis. Hum Mol Genet 18: 1578-1589, 2009.

22. Sharma LK, Fang H, Liu J, Vartak R, Deng J and Bai Y: Mitochondrial respiratory complex I dysfunction promotes tumorigenesis through ROS alteration and AKT activation. Hum Mol Genet 20: 4605-4616, 2011.

23. Shen L, Fan JG, Shao Y, Zeng MD, Wang JR, Luo GH, Li JQ and Chen SY: Prevalence of nonalcoholic fatty liver among administrative officers in Shanghai: An epidemiological survey. World J Gastroenterol 9: 1106-1110, 2003.

24. Zhou YJ, Li YY, Nie YQ, Ma JX, Lu LG, Shi SL, Chen MH and Hu PJ: Prevalence of fatty liver disease and its risk factors in the population of South China. World J Gastroenterol 13: 6419-6424, 2007.

25. Kim HY, Cho HK, Yoo SK and Cheong JH: Hepatic STAMP2 decreases hepatitis B virus $\mathrm{X}$ protein-associated metabolic deregulation. Exp Mol Med 44: 622-632, 2012.

26. Satapati S, Kucejova B, Duarte JA, Fletcher JA, Reynolds L, Sunny NE, He T, Nair LA, Livingston KA, Fu X, et al: Mitochondrial metabolism mediates oxidative stress and inflammation in fatty liver. J Clin Invest 125: 4447-4462, 2015.

27. Ringelhan M, O'Connor T, Protzer U and Heikenwalder M: The direct and indirect roles of HBV in liver cancer: Prospective markers for HCC screening and potential therapeutic targets. J Pathol 235: 355-367, 2015.

28. Ryerson AB, Eheman CR, Altekruse SF, Ward JW, Jemal A, Sherman RL, Henley SJ, Holtzman D, Lake A, Noone AM, et al: Annual report to the nation on the status of cancer, 1975-2012, featuring the increasing incidence of liver cancer. Cancer 122: 1312-1337, 2016.

29. Kumar R and Boon-Bee Goh G: Chronic hepatitis B and fatty liver: Issues in clinical management. Clin Res Hepatol Gastroenterol 40: 755-759, 2016.

30. Wang CC and Kao JH: Hepatitis B virus infection and decreased risk of nonalcoholic fatty liver disease: A cohort study. Hepatology 66: 681, 2017.

31. Wong VW, Wong GL, Chu WC, Chim AM, Ong A, Yeung DK, Yiu KK, Chu SH, Chan HY, Woo J, et al: Hepatitis B virus infection and fatty liver in the general population. J Hepatol 56: 533-540, 2012

32. Cheng YL, Wang YJ, Kao WY, Chen PH, Huo TI, Huang YH, Lan KH, Su CW, Chan WL, Lin HC, et al: Inverse association between hepatitis B virus infection and fatty liver disease: A large-scale study in populations seeking for check-up. PLoS One 8: e72049, 2013.

33. Wang Z, Xu M, Hu Z and Shrestha UK: Prevalence of nonalcoholic fatty liver disease and its metabolic risk factors in women of different ages and body mass index. Menopause 22: 667-673, 2015.

34. Wu YL, Peng XE, Zhu YB, Yan XL, Chen WN and Lin X: Hepatitis B Virus $X$ protein induces hepatic steatosis by enhancing the expression of liver fatty acid binding protein. J Virol 90: 1729-1740, 2015

35. Kim K, Kim KH, Kim HH and Cheong J: Hepatitis B virus X protein induces lipogenic transcription factor SREBP1 and fatty acid synthase through the activation of nuclear receptor LXRalpha. Biochem J 416: 219-230, 2008.

36. Nassir F and Ibdah JA: Role of mitochondria in nonalcoholic fatty liver disease. Int J Mol Sci 15: 8713-8742, 2014.

37. Liu L, Zhang K, Sandoval H, Yamamoto S, Jaiswal M, Sanz E, Li Z, Hui J, Graham BH, Quintana A and Bellen HJ: Glial lipid droplets and ROS induced by mitochondrial defects promote neurodegeneration. Cell 160: 177-190, 2015

38. Weinberg F, Hamanaka R, Wheaton WW, Weinberg S, Joseph J, Lopez M, Kalyanaraman B, Mutlu GM, Budinger GR and Chandel NS: Mitochondrial metabolism and ROS generation are essential for Kras-mediated tumorigenicity. Proc Natl Acad Sci USA 107: 8788-8793, 2010 International (CC BY-NC-ND 4.0) License. 